\title{
Autologous WT1-TCRc4 Gene-transduced CD8-positive Tcm/Tn Lymphocytes
}

National Cancer Institute

\section{Source}

National Cancer Institute. Autologous WT1-T CRC4 Gene-transduced CD8-positive

Tcm/Tn Lymphocytes. NCI Thesaurus. Code C121308.

Autologous, human CD8 T-lymphocytes, comprised of both central memory T-cells ( $\mathrm{T} \mathrm{cm}$ ) and naïve T-cells (T n), that are transduced, ex vivo, with a self-inactivating (SIN) lentiviral vector encoding a high-affinity T-cell receptor (TCRC4) specific for the human tumor antig en Wilms tumor 1 (WT 1) epitope 126-134 (RMFPNAPYL), with potential antineoplastic activity. Upon isolation of peripheral blood lymphocytes (PBLs), transduction, expansion ex vivo, priming of the Tn subset, but not the Tcm subset, with interleukin-21 (IL-21) and reintroduction of equal amounts of T cm and Tn cells into the patient, WT 1-TCRc4 gene-transduced CD8-positive T cm/T n lymphocytes redirect Tlymphocytes to WT 1-expressing tumor cells and specifically bind to and lyse those cells. This inhibits proliferation of WT 1-expressing tumor cells. WT1 protein, a zinc finger DNAbinding transcriptional regulator, is overexpressed in most leukemias and various solid tumors, while expression in normal, healthy tissues is very limited; its expression is correlated with aggressiveness and poor prognosis. 